\title{
Joint Range of Motion Decreased, CTCAE
}

National Cancer Institute

\section{Source}

National Cancer Institute. Joint Range of Motion Decreased, CT CAE. NCI Thesaurus.

Code C143629.

A disorder characterized by a decrease in joint flexibility of any joint. 